\title{
Autoimmune Polyglandular Syndrome Case Report Series
}

Raluca Nan*1, Cristian Guja ${ }^{1,2}$, Constantin Cucu ${ }^{1,3}$, Andra Caragheorgheopol ${ }^{1,3}$, Dana Manda ${ }^{3}$, Gabriela Radulian ${ }^{1,2}$, Mădălina Mușat ${ }^{\star 1,3}$

1 University of Medicine and Pharmacy „Carol Davila”, Bucharest Romania

${ }_{2}^{2}$ National Institute of Diabetes, Nutrition and Metabolic Disease "Prof. Dr. N. Paulescu",Bucharest Romania

${ }^{3}$ National Institute of Endocrinology "C.I. Parhon", Bucharest Romania

\section{Introduction}

The polyglandular autoimmune syndromes (PAS) are rare conditions characterized by the failure of several endocrine glands sometimes associated with other non-endocrine autoimmune diseases.There are four categories of PAS: PAS-I includes at least two out of: mucocutaneous candidiasis, hypoparathyroidism and adrenocortical failure. PAS-II comprises of Addison's disease, autoimmune thyroid disease and/or type 1 diabetes (Carpenter's syndrome). PAS-III is defined by the presence of autoimmune disorders other than Addison's disease and hypoparathyroidism, while PAS IV includes non-endocrine autoimmune disorders and Addison's disease, but not hypothyroidism.

\section{Case reports}

Case1 55-year-old woman

-Primary ovarian failure $(\mathrm{FSH}=16.26 \mathrm{mUl} / \mathrm{ml})$ at age of 33 .

-Addison's disease and chronic autoimmune thyroiditis at 42

9 a.m. plasma cortisol $=1.22 \mu \mathrm{g} / \mathrm{dl}, \mathrm{ACTH}=704.46 \mathrm{pg} / \mathrm{ml}$,

$\mathrm{TSH}=3.7 \mu \mathrm{lU} / \mathrm{ml}, \mathrm{T} 4=6.54 \mu \mathrm{g} / \mathrm{dl}, \mathrm{T} 3=117 \mathrm{ng} / \mathrm{dl}$, ATPO=1026 IU/L;

@46 years old: $\mathrm{TSH}=7.9 \mu \mathrm{lU} / \mathrm{ml}$

Thyroid ultrasound: diffuse, heterogeneous, hypoechogenicity, with increased blood flow.

Abdominal CT scan: hypoplastic adrenal glands -Diabetes mellitus

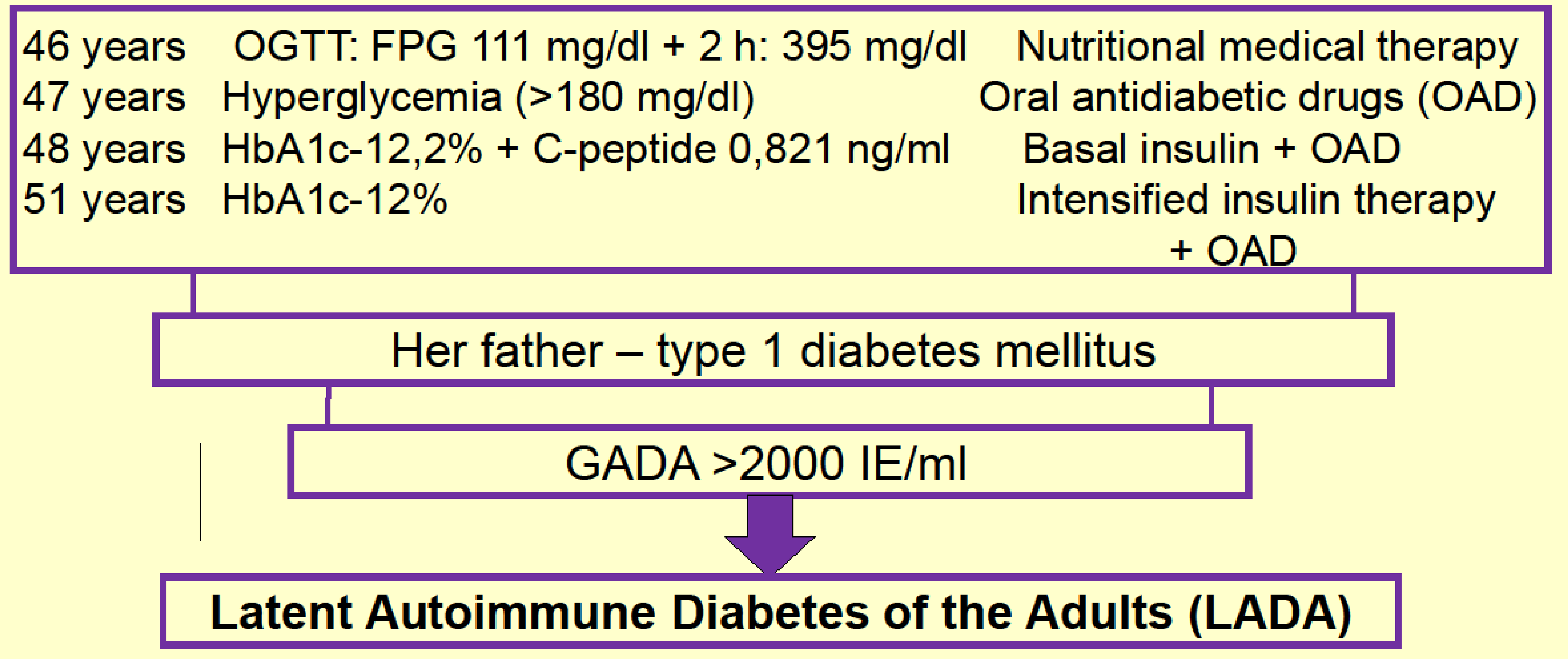

Currently on premixed insulin plus metformin $2.25 \mathrm{~g} /$ day, levothyroxine $75 \mathrm{mcg} / \mathrm{zi}$, hydrocortisone $25 \mathrm{mg} /$ day, fludrocortisone $0.1 \mathrm{mg} /$ day, statin HbA1c $7.0 \%$

Diagnoses: Polyglandular autoimmune syndrome type II: - Addison disease

- Chronic autoimmune thyroiditis

Latent autoimmune diabetes of the adults

- Primary ovarian failure

Case 242 old man

-vitiligo since 14 years old.

-@ 36 years Basedow's disease: muscle weakness, intense fatigue, sweating, tremor of the extremities, palpitations, weight loss. Labs: TSH $<0.03 \mu \mathrm{lU} / \mathrm{ml}(0.5-4.5 \mu \mathrm{Ul} / \mathrm{ml}), \mathrm{T} 4>20 \mu \mathrm{g} / \mathrm{dl}(4.5-13 \mu \mathrm{g} / \mathrm{dl}), \mathrm{T} 3>500$ $\mathrm{ng} / \mathrm{dl}$ (80-200 ng/dl). Thyroid ultrasound: diffuse hypoechogenicity, heterogeneous with increased vascularization.

\begin{tabular}{|c|c|c|}
\hline Pancreatic antibodies & Abnormal range & Normal range \\
\hline GADA & $>2000 \mathrm{IE} / \mathrm{ml}$ & $<10 \mathrm{IE} / \mathrm{ml}$ \\
\hline Pancreatic islet cell antibodies & $1 / 1000$ & $<1 / 10$ \\
\hline Anti-insulin antibodies & $10,9 \mathrm{U} / \mathrm{ml}$ & $<0,4 \mathrm{U} / \mathrm{ml}$ \\
\hline Anti-insulin receptor antibody & $\mathrm{IgG}=$ positive & \\
\hline
\end{tabular}

- @38 years old: Type 1 Diabetes mellitus: poliuro-polydipsia and weight loss (12 $\mathrm{Kg}$ in the last 5 months). HbA1c $9 \%$; basal bolus insulin therapy.

- @ 39 year-old total thyroidectomy + levothyroxine replacement.

- @ 40 year-old TSH=6.9 $\mu \mathrm{lU} / \mathrm{ml}, \mathrm{FT} 4=19.4 \mathrm{pmol} / \mathrm{l}, \mathrm{ACTH}=10.29$ $\mathrm{pg} / \mathrm{ml}(3-66 \mathrm{pg} / \mathrm{ml})$, cortisol= $12.78 \mu \mathrm{g} / \mathrm{dl}(6.7-22.6 \mu \mathrm{g} / \mathrm{dl})$, $\mathrm{HbA} 1 \mathrm{c}=9.67 \%$ (due to noncompliance with diet/ antiinsulin antibodies).

Currently on basal-bolus insulin therapy, levothyroxine $137.5 \mathrm{mcg} / \mathrm{day}$ after thyroidectomy.

Diagnoses: Polyglandular autoimmune syndrome type III: - Basedow's disease

- Type 1 diabetes mellitus - Vitiligo

Case 3 22-year-old woman

type 1 diabetes mellitus and primary hypothyroidism since she was 16 years old. ATPO $=0.18 \mathrm{IU} / \mathrm{ml}(<5.61 \mathrm{Ul} / \mathrm{ml})$.

-@17 years old: height $=150 \mathrm{~cm}$, weight $=36 \mathrm{Kg}, \mathrm{IMC}=16 \mathrm{Kg} / \mathrm{m}^{2}$

Labs: $\mathrm{HbA} 1 \mathrm{c}=4.81 \% \mathrm{TRA}=0.45 \mathrm{IU} / \mathrm{ml}, \mathrm{TSH}=4.3 \mu \mathrm{Ul} / \mathrm{ml}, \mathrm{FT} 4=18.2$ $\mathrm{pmol} / \mathrm{L}$, IGF-1=382 ng/ml (226-903 ng/ml).

Thyroid ultrasound: small goiter, diffuse hypoechogenicity

- @ 18 years old screening for other autoimmune diseases: estradiol = $110.91 \mathrm{pg} / \mathrm{ml}, \mathrm{LH}=6.73 \mathrm{mIU} / \mathrm{ml}, \mathrm{FSH}=8.46 \mathrm{mlU} / \mathrm{ml}$ - in normal range, $\mathrm{PRL}=7.48(2.8-29.2 \mathrm{ng} / \mathrm{ml}), 9$ a.m. plasma cortisol=20.09 (4.30-22.40 $\mu \mathrm{g} / \mathrm{dl}$ ), ATPO $<0.3 \mathrm{IU} / \mathrm{L}$ (ATPO may be absent in $10-15 \%$ of Hashimoto's disease, mostly in young).

Currently on basal-bolus insulin therapy and levothyroxine $50 \mathrm{mcg} / \mathrm{day}$.

Diagnoses: Polyglandular autoimmune syndrome:

- Type 1 diabetes mellitus

- Primary hypothyroidism

\section{Conclusion}

We emphasize the importance of screening for PAS after the first autoimmune disease is diagnosed. The key to successfully managing patients with PAS is to identify and treat their disorders early before complications occur. This may be achieved by early screening for autoantibodies or subclinical endocrine failure. Patients should be educated to comply with the lifelong medical surveillance and encourage their family members to be screened for autoimmune diseases as about $50 \%$ of patients with PAI II have siblings with autoimmune diseases.

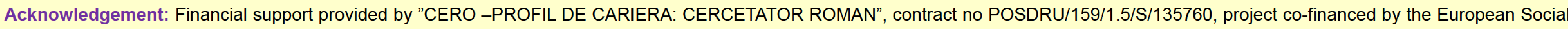
Fund through Sectoral Operational Programme Human Resources Development 2007-2013.

*These authors have contributed equally to this study. 